\title{
ACEITABILIDADE E INTENÇÃO DE COMPRA DO QUEIJO DE COALHO DE CABRA TEMPERADO COM CACHAÇA
}

\author{
Acceptability and purchase intention of goat's Coalho cheese \\ seasoned with cachaça
}

Vanessa Bonfim da Silva ${ }^{*}$, Marion Pereira da Costa ${ }^{I}$, Nelson de Carvalho Delfino ${ }^{l}$

\section{RESUMO}

O leite de cabra apresenta melhor digestibilidade e hipoalergenicidade quando comparado ao leite bovino. Nesta perspectiva, o leite caprino apresenta elevado potencial na elaboração de derivados lácteos, principalmente queijos, sendo considerado um excelente substituto ao leite bovino na alimentação humana. Um dos queijos mais produzidos a partir do leite de cabra é o queijo de coalho, cuja exploração de novos condimentos torna-se interessante a fim de valorizá-lo culturalmente e economicamente no mercado. Por esses motivos, o objetivo deste trabalho foi elaborar um novo queijo de coalho de cabra utilizando a cachaça como condimento, além de verificar a aceitação (aceitação global, sabor, aroma, aparência e textura) e intenção de compra deste novo produto em uma população potencialmente consumidora. Quanto a aceitação global, os valores médios variaram de 6,88 $\pm 1,53 \mathrm{a}$ 7,51 $\pm 1,18$ com conceito médio "gostei moderadamente". Estes resultados também se mostram promissores quando avaliada a aceitabilidade dos atributos sensoriais em valores absolutos e percentuais. A aparência e a textura foram os atributos que mais se destacaram positivamente, obtendo ambos o conceito "gostei muito", com valores médios de 7,51 $\pm 1,18$ e 7,35 $\pm 1,35$, respectivamente. As características sabor e aroma variaram entre os conceitos "gostei moderamente" e "gostei muito", com notas variando de 6,88 $\pm 1,53$ e 6,89 $\pm 1,63$,respectivamente. Ademais, 76,3\% dos julgadores afirmaram que comprariam o queijo de coalho caprino temperado com cachaça. Desta forma, o queijo elaborado obteve boa aceitabilidade e intenção de compra, indicando que a cachaça pode ser utilizada como condimentação de queijo de coalho caprino, aumentando a atratividade e visibilidade do produto.

Palavras-chave: leite de cabra; queijo condimentado; aceitação global.

1 Universidade Federal da Bahia (UFBA), Escola de Medicina Veterinária e Zootecnia (EMEVZ), Av. Adhemar de Barros, 500, Ondina, 40170-110, Salvador, BA, Brasil. E-mail: vbonfim90@gmail.com.

* Autor para correspondência.

Recebido / Received: 16/02/2017

Aprovado / Approved: 08/12/2017 


\begin{abstract}
Goat milk has better digestibility, high protein content and hypoallergenicity when compared to bovine milk. From this point of view, goat milk has high potential in the elaboration of dairy products, particularly cheese, and is considered an excellent substitute for cow's milk for human consumption. One of the cheeses most produced using goat's milk is coalho cheese, in which the use of new condiments becomes interesting to value it culturally and economically on the market. For these reasons, the objective of this work was to elaborate a goat's Coalho cheese with cachaça, besides to verify the acceptance (global acceptance, flavor, aroma, appearance and texture) and purchase intention in a population potentially consumer. Regarding the global acceptance, mean values ranged from $6,88 \pm 1,53$ to $7,51 \pm 1,18$ with a concept "I liked moderately". These results are also promising when evaluating the acceptability of the sensorial attributes in absolute and percentage values. The appearance and texture were the attributes that most stood out positively, obtaining both the concept "I liked very much", with mean values of 7,51 $\pm 1,18$ and 7,35 $\pm 1,35$, respectively. The flavor and aroma varied between concepts "I liked moderately" and "I liked very much", with grades ranging from $6,88 \pm 1,53$ and 6,89 $\pm 1,63$, respectively. In addition, $76,3 \%$ of the judges affirmed that they would buy the goat cheese seasoned with cachaça. Thus, the elaborated cheese obtained good acceptability and purchase intention, indicating that the cachaça can be used as condiment of caprine coalho cheese, increasing its attractiveness and visibility.
\end{abstract}

Keywords: goat milk; seasoned cheese; global acceptance.

\section{INTRODUÇÃO}

Em 2014, o Brasil produziu 153.659 toneladas de leite de cabra, apresentando crescimento significativo de 1961 a 2014 (FAO, 2014). Desta forma, a caprinocultura leiteira brasileira tem superado os constantes desafios, mantendo e conquistando novos nichos de mercados para o leite de cabra e seus derivados (SILVA et al., 2012). A região Nordeste se destaca, detendo $92 \%$ do rebanho caprino brasileiro (IBGE, 2014). No entanto, nesta região o principal beneficiamento do leite de cabra é a pasteurização direcionando o leite pasteurizado para programas governamentais que o redistribuem em escolas públicas (PERDIGÃO et al., 2016). Portanto, a industrialização do leite de cabra ainda é restrita no país, principalmente na região Nordeste, devido não só à pequena produção individual, hábito alimentar da população, diversidade de produtos lácteos ofertados desta matriz, desconhecimento dos valores nutricionais, mas também pelo preconceito e alto preço comparado aos similares bovino (GARCIA; TRAVASSOS, 2012).

O leite de cabra, quando comparado ao leite de vaca, apresenta melhor digestibilidade, teor de proteínas de alto valor nutricional e hipoalergenicidade (SANTOS et al., 2012), entre outros. Estas características nutricionais e terapêuticas o tornam um excelente substituto ao leite bovino na nutrição humana, principalmente para indivíduos alérgicos ao leite de vaca, idosos e crianças (CENACHI et al., 2011;GARCIA; TRAVASSOS, 2012; SOUZA et al., 2013). Contudo, esta matriz láctea apresenta uma elevada concentração de ácidos graxos de cadeia curta, representados pelos ácidos caprílico, 
cáprico e capróico (CEBALLOS et al., 2009; PARK et al., 2007), que influenciam de forma negativa na aceitação de seus derivados frente a consumidores não habituais (COSTA et al., 2014; COSTA et al., 2015). Por outro lado, Correia; Borges (2009), avaliando a intenção de compra do leite de cabra e seus derivados na cidade de Natal-RN, observaram que existe uma predisposição ao consumo de derivados de leite de cabra, sobretudo o queijo caprino.

O queijo de coalho é definido como “o queijo que se obtém por coagulação do leite por meio do coalho ou outras enzimas coagulantes apropriadas, complementada ou não pela ação de bactérias lácteas selecionadas e comercializado normalmente com até 10 (dez) dias de fabricação" (BRASIL, 2001). No Brasil, este queijo, tradicionalmente nordestino, é um dos queijos mais produzidos a partir do leite de cabra (ANDRADE, 2006; MAMEDE et al., 2010; OLIVEIRA et al., 2012; SANTOS et al., 2012; QUEIROGA et al., 2013; SOUSA et al., 2014), sendo uma importante atividade no âmbito social e econômico da região (GARCIA et al., 2008; CORREIA; BORGES, 2009). Portanto, torna-se interessante a maior diversidade destes produtos por meio da adição de novos condimentos, a fim de aumentar a valorização do queijo de coalho, oferecendo qualidade e ampliando a oferta do derivado no comércio.

Nesta conjuntura, a cachaça pode ser utilizada como um condimento para valorizar o queijo de coalho caprino, pois ambos os produtos são exclusivamente brasileiros, os quais possibilitariam a valorização de produtos regionais e o fortalecimento de ambos os setores na região Nordeste. Esta região apresenta vários produtores de cachaça os quais localizam-se, principalmente, nos estados de Pernambuco, Ceará, Paraíba, Alagoas, Rio Grande do Norte e Bahia (LIMA, 2006). A cachaça ainda é a segunda bebida mais vendida no Brasil, perdendo apenas para a cerveja e é a bebida destilada mais popular do Brasil (VILA NOVA, 2008). Além disso, não há registros na literatura sobre queijo temperado com cachaça, sendo este o primeiro relato de sua elaboração.

Por estes motivos, o objetivo deste trabalho foi elaborar um queijo de coalho a partir do leite de cabra temperado com cachaça, bem como verificar sua aceitação e intenção de compra em uma população potencialmente consumidora.

\section{MATERIAL E MÉTODOS}

\section{Obtenção da matéria-prima}

O leite de cabra utilizado para elaboração do queijo foi oriundo da Fazenda Experimental da UFBA no município de Entre Rios, Bahia, pertencente à Escola de Medicina Veterinária e Zootecnia (EMEVZ) da Universidade Federal da Bahia. O material foi acondicionado em galões plásticos específicos dentro de isopor com gelo e mantidos sob refrigeração em temperatura não superior a $7{ }^{\circ} \mathrm{C}$ e transportado imediatamente para o Laboratório de Inspeção e Tecnologia de Leite e Derivados (LaITLácteos) na EMEVZ.

\section{Elaboração do queijo de coalho de cabra}

O leite de cabra (10 litros) foi pasteurizado $\left(65^{\circ} \mathrm{C} / 30 \mathrm{~min}\right)$ e, em seguida, resfriado à $35^{\circ} \mathrm{C}$. Após o tratamento térmico, foram adicionado os ingredientes na seguinte ordem: $1 \%$ de fermento (Streptococcus thermophilus e Lactobacillus delbrueckii bulgaricus; Iogurte Natural Desnatado Nestlé ${ }^{\circledR}$ ), 0,05\% de cloreto de cálcio (Rica Nata cód. 1059), 0,005\% de coagulante líquido (CHY - MAX ${ }^{\circledR}$ EXTRA; quimosina microbiana, Aspergillus niger var. awamor; cód. GIN:674918), mexendo por 2 min após a adição de cada ingrediente. Em seguida, a massa foi deixada em repouso por 50 minutos, realizou-se após esse período o corte da coalhada em cubos e a massa 
descansou por mais 5 min. Depois, agitou-se lentamente a massa por 20 min e novamente descansou-se mais 5 min. Retirou-se $50 \%$ do soro para aquecê-lo a $70{ }^{\circ} \mathrm{C}$ e retorná-lo ao queijo lentamente até chegar a $55^{\circ} \mathrm{C}$. Realizou-se a última mexedura por 50 minutos e posterior dessoragem. Adicionou-se 1,5\% de cloreto de sódio. Em seguida o queijo foi enformado, prensado e deixado em repouso por $12 \mathrm{~h}$. No dia seguinte, mergulhou-se o queijo de coalho na cachaça ouro (registro do produto no MAPA n ${ }^{\circ} \mathrm{CE}$ - 05500 00061-3) por $12 \mathrm{~h}$. Após esse período, embalou-se o queijo em sacos plásticos de polietileno e realizou-se a maturação a temperatura de $10{ }^{\circ} \mathrm{C}$ por 10 dias.

O queijo de coalho elaborado foi submetido às análises microbiológicas para verificação de sua segurança alimentar para posterior realização da análise sensorial. Apresentando-se o queijo de coalho elaborado dentro dos parâmetros preconizados pela Instrução Normativa $n^{\circ} 62$, de 26 de agosto de 2003, pode-se realizar a análise sensorial (BRASIL, 2003).

\section{Análise Sensorial}

A análise sensorial foi realizada na Escola de Medicina Veterinária e Zootecnia da Universidade Federal da Bahia (EMEVZ/ UFBA). Para formar o painel sensorial foram recrutados 80 julgadores não treinados, entre os alunos e servidores da UFBA por meio de um questionário (Figura 1), aplicando-se o teste de aceitação utilizando a escala hedônica estruturada em nove (9) pontos, com escore variando de um (1) "desgostei extremamente" até nove (9) "gostei extremamente", com base nos atributos sabor, aroma, aparência, textura e conjunto (aceitação global). Ainda neste questionário, foram aplicadas questões de resposta única (sim ou não) para os julgadores sobre: já ter consumido ou não leite de cabra ou algum derivado; conhecimento da amostra em estudo e intenção de compra. Nesta última, em caso de resposta negativa, foi perguntado o motivo mais forte para não ter a intenção de compra e dadas as opções dos atributos da amostra (sabor, aroma, aparência, textura, outros).

Os provadores foram separados em cabines individuais, condições de luminosidade branca controlada, com temperatura de $20{ }^{\circ} \mathrm{C}$ e ausência de ruídos e aromas que interferissem na análise.

\section{Análise de Resultados}

Para análise de resultados, os dados foram organizados em planilha eletrônica do Microsoft Office Excel ${ }^{\circledR}$ e foram utilizadas ferramentas da análise estatística descritiva, através da qual foram mostrados os valores absolutos e percentuais obtidos. Os dados foram organizados na forma de tabela e gráficos, considerando os vários atributos: sabor, aroma, aparência, textura e aceitação global.

\section{RESULTADOS E DISCUSSÃO}

Quanto aos provadores $(\mathrm{n}=80 ; 50 \mathrm{mu}-$ lheres, 30 homens), cerca de $62,5 \%$ eram mulheres e $37,5 \%$ homens, com idade variando de 18 a 63 anos, dos quais $68,8 \%$ possuíam idade entre 20 e 30 anos, seguido de 13,8\% entre 30 e 40 anos. A maioria dos julgadores afirmou possuir nível superior incompleto (65\%), seguido de $12,5 \%$, que afirmaram possuir pós-graduação.

\section{Teste de Aceitabilidade}

Os resultados do teste de aceitabilidade dos atributos sensoriais relacionados ao teste de aceitação do queijo de coalho de cabra temperado com cachaça são apresentados na Tabela 1. 


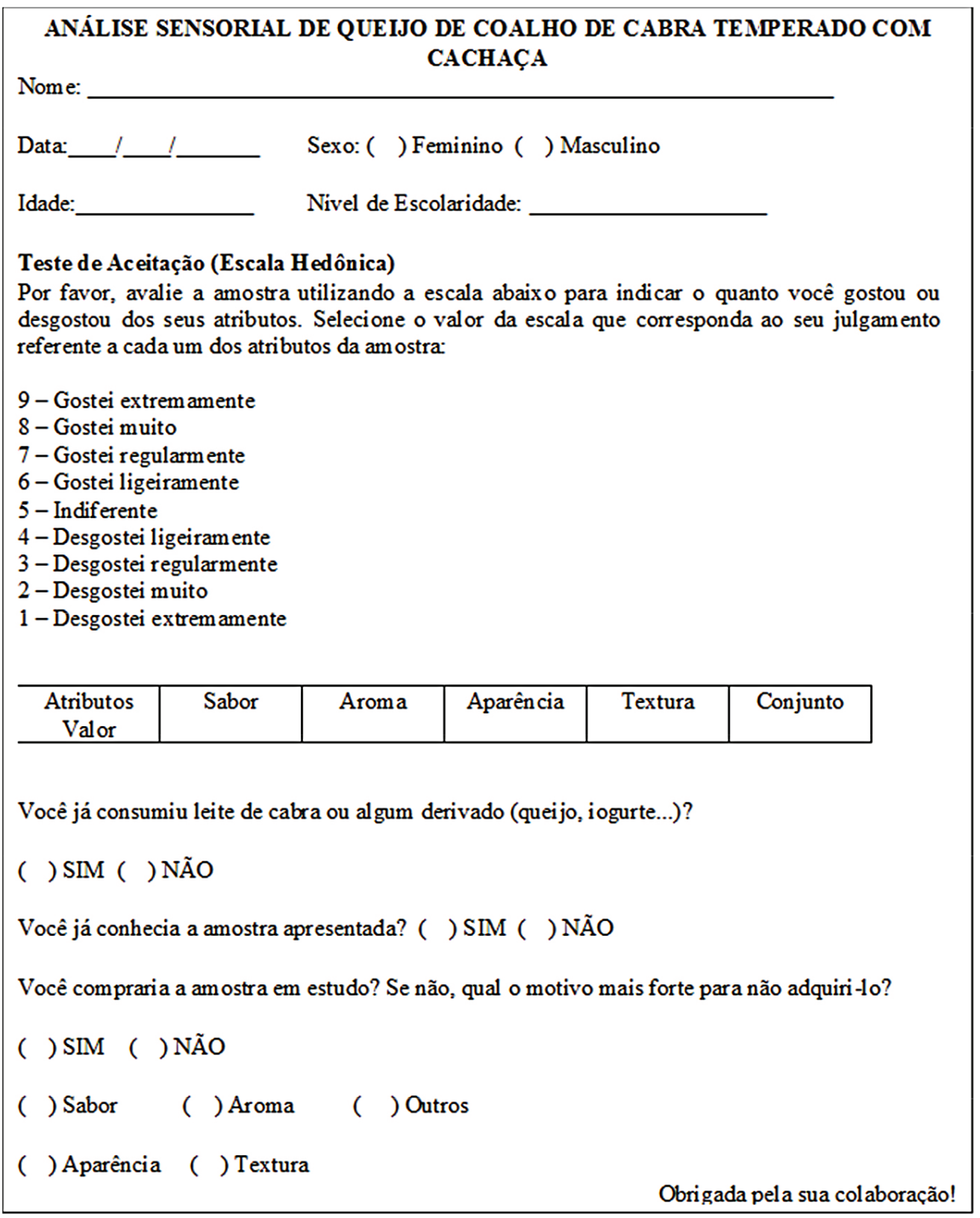

Figura 1 - Questionário aplicado para realização da análise sensorial 
Os valores médios obtidos no teste de aceitação $(6,88$ - 7,51) estão de acordo com a escala hedônica, no conceito "gostei moderadamente". Souza et al. (2011), em estudo com queijo de coalho de cabra condimentado com cumaru, obtiveram valores semelhantes aos do presente estudo para o teste de aceitação $(6,93$ - 7,23), o qual também consistiu no conceito "gostei moderadamente" na escala hedônica de nove pontos. Contudo, em comparação com o trabalho de Curi; Bonassi (2007), os quais realizaram análise sensorial do queijo análogo ao pecorino romano produzido com leite de cabra e coalhada congelados, os resultados deste estudo se mostraram superiores, sendo assim pode-se inferir que o tipo de queijo e sua condimentação interfere na aceitação do consumidor pelo produto, reforçando que o queijo de coalho de cabra temperado com cachaça conseguiu atingir uma aceitação que pode ser promissora para sua comercialização.

Estes resultados também se mostraram promissores quando avaliada a aceitabilidade dos atributos sensoriais em valores absolutos e percentuais (Figuras 2 e 3 ).

As características sabor e aroma variaram em sua maioria entre o "gostei moderamente" e "gostei muito". Entretanto, apesar da média obtida nos testes de aceitação, analisando os valores absolutos para a variação na

Tabela 1 - Escores de atributos sensoriais do queijo de coalho de cabra temperado com cachaça

\begin{tabular}{lc}
\hline \multicolumn{1}{c}{ Atributo } & $\begin{array}{c}\text { Score } \\
\text { (Valor médio } \pm \text { desvio padrão) }\end{array}$ \\
\hline Sabor & $6,88 \pm 1,53$ \\
Aroma & $6,89 \pm 1,63$ \\
Aparência & $7,51 \pm 1,18$ \\
Textura & $7,35 \pm 1,35$ \\
Aceitação global & $7,19 \pm 1,30$ \\
\hline
\end{tabular}

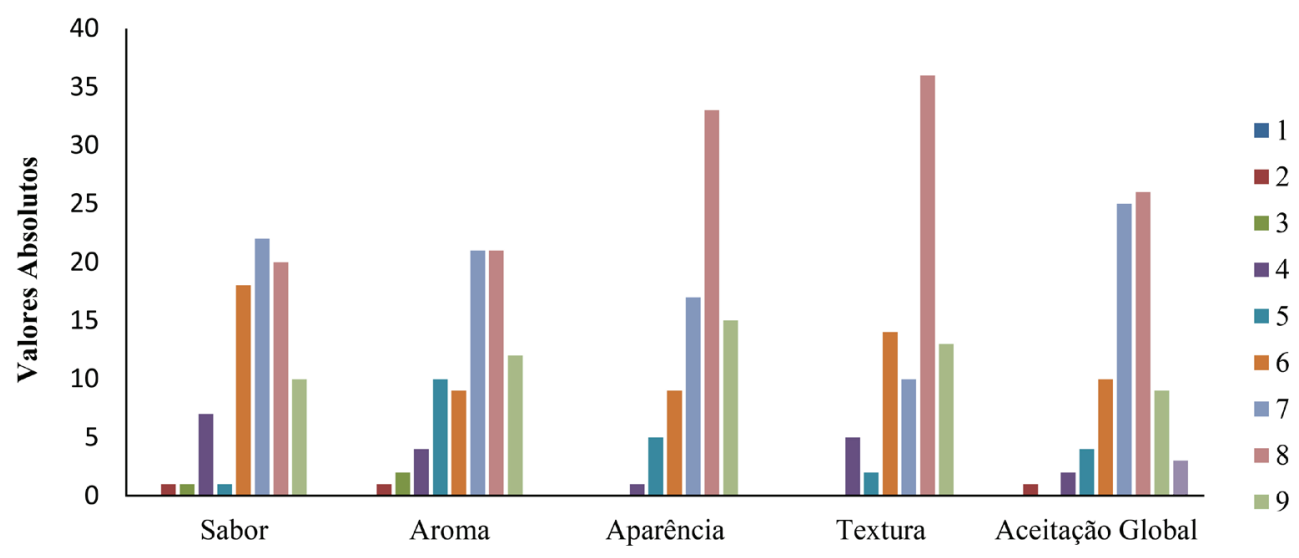

Atributos Sensoriais

Figura 2 - Gráfico da aceitabilidade dos atributos sensoriais do queijo de coalho de cabra temperado com cachaça expresso em valores absolutos 


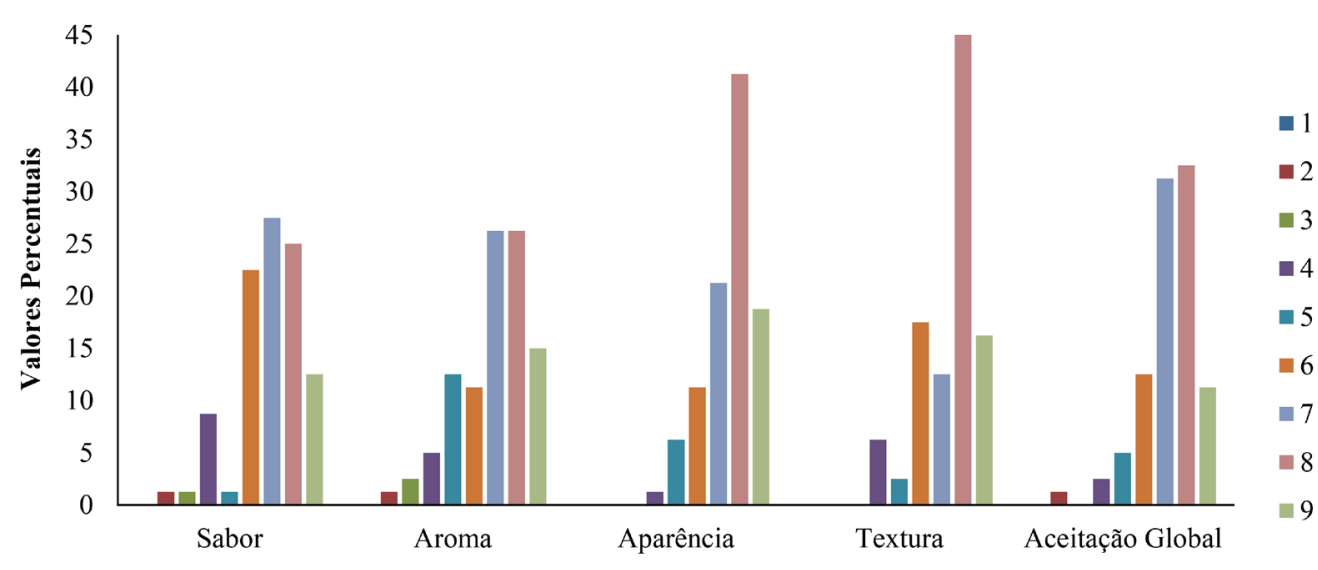

Atributos Sensoriais

Figura 3 - Gráfico da aceitabilidade dos atributos sensoriais do queijo de coalho de cabra temperado com cachaça expresso em percentuais (\%)

escala hedônica entre o "gostei ligeiramente" e "gostei extremamente". Pode-se perceber que, 70 degustadores $(87,5 \%)$ responderam que gostaram do sabor e $63(78,8 \%)$ gostaram do aroma. Segundo Garcia et al. (2008), esse comportamento pode ser explicado pelo fato dos julgadores estarem habituados a consumir produtos elaborados com leite de vaca.

Para Garcia; Travassos (2012), o leite de cabra apresenta características sensoriais peculiares, sobretudo nos atributos cor, sabor e aroma, as quais podem comprometer a aceitabilidade dos produtos. Segundo Guiné et al. (2015), os queijos obtidos a partir de leite cru apresentam um desenvolvimento de aromas mais intenso, mais rápido e mais completo, bem como uma textura mais macia, resultante da extensão da proteólise, que origina peptídeos e aminoácidos, os quais influenciam consideravelmente as características sensoriais do queijo. Apesar da legislação não permitir, a fabricação artesanal utiliza predominantemente o leite cru, o qual confere ao queijo características únicas percebidas pelo consumidor. Já industrialmente, o leite utilizado para a produção de queijo é o leite pasteurizado e ainda há a adição de culturas lácteas, o que em conjunto levam a alteração nas características sensoriais e percepção do consumidor (ANDRADE, 2006).

A aparência e a textura são os atributos que mais se destacaram positivamente, obtendo ambos, em sua maioria, conceito "gostei muito" na escala hedônica. Considerando a variação na escala hedônica entre o "gostei ligeiramente" e "gostei extremamente", dos 80 degustadores, $74(92,5 \%)$ afirmaram que gostaram da aparência e 73 (91,3\%) afirmaram que gostaram da textura. Em relação à textura, o conceito aqui observado é muito superior ao encontrado por Curi; Bonassi (2007), quando eles avaliaram o queijo análogo ao pecorino romano de cabra.

Em relação a aceitabilidade global, esta obteve resultado mais expressivo na escala hedônica entre o "gostei moderadamente" e "gostei muito", muito provavelmente influenciada pelo sabor e aroma do produto. Contudo, quando se analisa os valores absolutos e percentuais, percebe-se que 73 julgadores $(87,5 \%)$ gostaram do queijo, sendo que destes $51(63,8 \%)$ gostaram moderadamente ou muito. 


\section{Consumo de leite de cabra, conhecimento da amostra e intenção de compra}

A maioria $(67,5 \%)$ afirmou já ter consumido leite de cabra ou algum derivado lácteo caprino e apenas 3,75\% afirmaram conhecer a amostra apresentada, uma vez que esta já se encontra presente no mercado para comercialização.

Sobre a intenção de compra, 76,3\% após ter consumido a amostra afirmaram que comprariam o queijo de coalho de cabra temperado com cachaça, sendo que destes, $55,7 \%$ eram mulheres e $44,3 \%$ eram homens, evidenciando que a intenção de compra é aproximadamente semelhante entre homens e mulheres, considerando o percentual de participação na análise sensorial. Este resultado corrobora com o estudo de Souza et al. (2011), no qual observaram que 68\% dos julgadores afirmaram que comprariam o queijo de coalho de cabra condimentado com cumaru. Ainda, este resultado está em acordo com o resultado de Correia; Borges (2009), que observaram que o queijo é o derivado lácteo caprino com maior pré-disposição para compra e ainda, dentro dos produtos incluídos na pesquisa, eles afirmam que o queijo é o derivado de maior penetração comercial. O percentual de intenção de compra mostrou que existe pré-disposição ao consumo do queijo de cabra, o que incentiva bastante sua comercialização.

O queijo é referido na literatura como o produto de maior interesse tecnológico e econômico produzido com o leite de cabra, sobretudo na Europa (CURI; BONASSI, 2007; KATIKI et al., 2009). Esse poderia ser um mercado para promover a caprinocultura leiteira no país, visto que o Brasil poderia se tornar a longo prazo exportador para países europeus, que são reconhecidamente grandes consumidores de queijo de cabra, como França, Espanha, Itália e Grécia (CURI; BONASSI, 2007; GARCIA et al., 2008; KATIKI et al., 2009; GARCIA; TRAVASSOS,
2012). Nesta perspectiva, ainda o queijo nacional produzido poderia substituir os queijos importados, os quais possuem preços elevados, e assim também incentivar o a produção caprina leiteira (SOUZA et al., 2008).

Ao ser questionado o motivo mais forte para não adquirir o produto, $82,3 \%$ dos julgadores responderam que o principal aspecto negativo foi o sabor, seguido do aroma $(11,8 \%)$ e outros $(5,9 \%)$. Estes resultados corroboram com a literatura que evidencia que os atributos sensoriais decisivos para não comprar leite de cabra e derivados, são o aroma e o sabor (CORREIA; BORGES, 2009; COSTA et al., 2013, COSTA et al., 2014; COSTA et al., 2015). Segundo Garcia et al. (2008), em análise sensorial de produtos elaborados com leite caprino utilizando uma sequência de amostras e uma ficha de avaliação contendo uma escala hedônica estruturada de nove pontos, os provadores perceberam diferença no aroma e no sabor dos produtos apresentados.

\section{CONCLUSÕES}

O queijo de coalho de cabra temperado com cachaça obteve boa aceitabilidade. Com os resultados obtidos, pode-se inferir que o desenvolvimento de um novo derivado lácteo caprino pode aumentar o consumo dos produtos caprinos. A adição de condimentação a um queijo tão popular quanto o queijo de coalho aumenta a atratividade do produto, incentivando ainda mais o mercado leiteiro caprino.

\section{AGRADECIMENTOS}

À Fazenda Experimental da Universidade Federal da Bahia no município de Entre Rios, Bahia, por ceder o leite de cabra necessário para a elaboração do queijo. Aos técnicos do Laboratório de Inspeção e Tecnologia de Leite e Derivados da Escola de Medicina Veterinária e Zootecnia (EMEVZ) 
da Universidade Federal da Bahia, Jaqueline Alcântara e Davino Alves, pela disponibilidade e auxílio para realização deste estudo.

\section{REFERÊNCIAS}

ANDRADE, A. A. Estudo do perfil sensorial, físico-químico e aceitação de queijo de coalho produzido no Estado do Ceará. 2006. 127p. Dissertação (Mestrado em Tecnologia de Alimentos) - Universidade Federal do Ceará, Ceará, 2006.

BRASIL. Ministério da Agricultura, Pecuária e Abastecimento. Instrução Normativa $\mathrm{n}^{\circ} 30$, de 26 de junho de 2001. Regulamento Técnico de Identidade e Qualidade de Queijo de Coalho. Diário Oficial da República Federativa do Brasil, Brasília, 16 jul. 2001.

BRASIL. Ministério da Agricultura, Pecuária e Abastecimento. Departamento de Inspeção de Produtos de Origem Animal. Instrução Normativa $n^{0} 62$, de 26 de agosto de 2003. Oficializa os métodos analíticos oficiais para análises microbiológicas para controle de produtos de origem animal e água. Diário Oficial da República Federativa do Brasil, Brasília, 18 set. 2003. Seção 1, p. 14.

CEBAllos, L. S. et al. Composition of goat and cow milk produced under similar conditions and analyzed by identical methodology. Journal of Food Composition and Analysis, v. 22, n. 4, p. 322-329, 2009.

CENACHI, D. B. et al. Aspectos composicionais, propriedades funcionais, nutricionais e sensoriais do leite de cabra: uma revisão. Revista do Instituto de Laticínios Cândido Tostes, v. 66, n. 382, p. 12-20, 2011.

CORREIA, R. T. P.; BORGES, K. C. Posicionamento do consumidor frente ao consumo de leite de cabra e seus derivados na cidade de Natal-RN. Revista do Instituto de Laticínios Cândido Tostes, v. 64, n. 366, p. 36-43, 2009.

COSTA, M. P. et al. Leite fermentado: potencial alimento funcional. Enciclopédia Biosfera, v. 9, n. 16, p. 1387-1408, 2013.

COSTA, M. P. et al. Changes in expected taste perception of probiotic and conventional yogurts made from goat milk after rapidly repeated exposure. Journal of Dairy Science, v. 97 , n. 5, p. 2610-2618, 2014.

COSTA, M. P. et al. Determination of biogenic amines by high-performance liquid chromatography (HPLC-DAD) in probiotic cow's and goat's fermented milks and acceptance. Food Science \& Nutrition, v. 3, n. 3, p. 172-178, 2015.

CURI, R.; BONASSI, I. Elaboração de um queijo análogo ao Pecorino Romano produzido com leite de cabra e coalhada congelados. Ciência e Agrotecnologia, Lavras, v. 31, n. 1, p. 171-176, 2007.

FAO - FOOD AND AGRICULTURE ORGANIZATION. FAOSTAT, 2014. Disponível em: $<$ http://www.fao.org/faostat/en/\#data/QL $>$. Acesso em: 27 jun. 2017.

GARCIA, R. V. et al. Aceitabilidade e preferência sensorial do queijo de coalho de leite búfala, de leite cabra e de leite de vaca. Revista do Instituto de Laticínios Cândido Tostes, v. 63, n. 363, p. 12-16, 2008.

GARCIA, R. V.; TRAVASSOS, A. E. R. Aspectos gerais sobre o leite de cabra: uma revisão. Revista do Instituto de Laticínios Cândido Tostes, v. 67, n. 386, p. 81-88, 2012.

GUINÉ, R. et al. Avaliação comparativa de queijos portugueses de cabra e ovelha. Millenium, v. 49, p. 111-130, 2015.

IBGE. Instituto Brasileiro de Geografia e 
Estatística. Cidades. 2014. Disponível em: $<$ http://www.ibge.gov.br/home/estatistica/ economia/ppm/2014/default.shtm $>$. Acesso em: 17 jul. 2016.

KATIKI, L. M. et al. Aspectos físico-quími$\cos$ e microbianos do queijo maturado por mofo obtido da coagulação mista com leite de cabra congelado e coalhada congelada. Ciência e Tecnologia de Alimentos, v. 26, n. 4, p. 740-743, 2006.

LIMA, J. P. R. Cachaça artesanal e vinhos finos no Nordeste: desafios, potencialidades e indicações políticas. Revista Econômica do Nordeste, v. 37, n. 4, p. 609-628, 2006.

MAMEDE, M. E. O. et al. Estudo das características sensoriais e da composição química de queijo de coalho industrializado. Revista do Instituto Adolfo Lutz, v. 69, n. 3, p. 364-70, 2010.

OLIVEIRA, M. E. G. et al. Technological, physico chemical and sensory characteristics of a Brazilian semi-hard go at cheese (coalho) with ad ded probiotic lactic acid bacteria. Scientia Agricola, v. 69, n. 6, p. 370379, 2012.

PARK, Y. W. et al. Physico-chemical characteristics of goat and sheep milk. Small Ruminant Research, v. 68, n. 1-2, p. 88-113, 2007.

PERDIGÃO, N. R. O. F. et al. Sistemas de Produção de Caprinos Leiteiros. In: WORKSHOP SOBRE PRODUÇÃO DE CAPRINOS NA REGIÃO DA MATA ATLÂNTICA, 13., 2016, Coronel Pacheco. Anais eletrônicos... Coronel Pacheco: Embrapa Gado de Leite, 2016. Disponível em: $<$ https://www.alice.cnptia. embrapa.br/alice/bitstream/doc/1051053/1/ CNPC2016Doc119.pdf>. Acesso em: 29 jun. 2017.

QUEIROGA, R. C. R. E. et al. Nutritional, textura land sensory properties of Coalho cheese made of goats', cows' milk and their mixture. Food Science and Technology, v. 50, p. $538-544,2013$.

SANTOS K. M. O. et al. Probiotic caprine Coalho cheese natural yenriched in conjugated linoleic acid as a vehicle for Lactobacillus acido philus and beneficial fatty acids. International Dairy Journal, v. 24, p. 107112, 2012.

SILVA, H. W. et al. Aspectos da exploração da caprinocultura leiteira no Brasil. Revista Brasileira de Agropecuária Sustentável (RBAS), v. 2, n. 2, p. 121-125, 2012.

SOUSA, A. Z. B. et al. Aspectos físico-químicos e microbiológicos do queijo tipo coalho comercializado em estados do nordeste do Brasil. Arquivos do Instituto Biológico, v. 81, n. 1, p. 30-35, 2014.

SOUZA, V. L. F. et al. Produção de queijo de leite de cabra, tipo Camponês, com diferentes níveis de fermento lático. Revista Brasileira de Saúde e Produção Animal, v. 9, n. 2, p. 334-340, 2008.

SOUZA, E. L. et al. Qualidade do queijo de leite de cabra tipo Coalho condimentado com cumaru (Amburana cearensis A. C. Smith). Brazilian Journal of Food Technology, v. 14, n. 3, p. 220-225, 2011.

SOUZA, A. K. et al. Características microbiológicas e físico-químicas do leite de cabra submetido à pasteurização e ao congelamento, comercializado na cidade de Alfenas-MG. Revista da Universidade Vale do Rio Verde, v. 11, n. 1, p. 224-233, 2013.

VILA NOVA, M. X. Análise de leveduras do mosto da fermentação alcoólica de alambiques artesanais produtores de cachaça em Pernambuco. 2008. 118 f. Tese (Doutorado em Ciências Biológicas) - Universidade Federal de Pernambuco, Recife, 2008. 